Research Article

\title{
Stability Optimization of Trapezoidal Frame with Rigid Members and Flexible Joints
}

\author{
Chao Wu ${ }^{1},{ }^{1}$ Ya-Nan Li, ${ }^{2}$ Lik-Ho Tam, ${ }^{1}$ and Li He ${ }^{3}{ }^{3}$ \\ ${ }^{1}$ School of Transportation Science and Engineering, Beihang University, 37 Xueyuan Road, Beijing 100191, China \\ ${ }^{2}$ School of Energy and Power Engineering, Beihang University, 37 Xueyuan Road, Beijing 100191, China \\ ${ }^{3}$ Department of Environmental Science and Engineering, School of Light Industry, Beijing Technology and Business University, \\ Beijing 100048, China \\ Correspondence should be addressed to Li He; he.li@btbu.edu.cn
}

Received 23 April 2020; Revised 30 April 2020; Accepted 20 May 2020; Published 2 June 2020

Academic Editor: Michael Yam

Copyright (c) 2020 Chao Wu et al. This is an open access article distributed under the Creative Commons Attribution License, which permits unrestricted use, distribution, and reproduction in any medium, provided the original work is properly cited.

\begin{abstract}
Stability has been an important subject in the design of a portal frame structure. Conventional stability analysis of the portal frame is normally conducted assuming that all the joints are rigid. However, the joints of a portal frame in real applications are not always rigid and semirigid connections often exist. AISC design code requires that the effect of the joint flexibility on the behavior and buckling capacity of the portal frame should be taken into account in the analysis and design procedures. To address this issue, a portal frame with flexible joints and rigid members was theoretically analyzed in the literature and closed form solution was derived for its global buckling load. However, when more parameters are involved, e.g., different leg lengths, asymmetric frame shape, and moving load, the solution to the governing equation of the stability of the frame becomes impossible. This paper presents a comprehensive parametric study on the stability of an asymmetric portal frame with flexible joints and rigid members through finite element (FE) analysis. The FE model was first validated by the existing theoretical solution in the literature. Parameters including the position of the moving load, the lengths of the two frame legs, and the span of the frame were analyzed. Design curves were developed based on the parametric study, from which the stable, unstable, and catastrophically unstable states of the portal frame were characterized. This paper contributes benchmark results for the stability optimization in the design of the portable frame of a general shape.
\end{abstract}

\section{Introduction}

Stability has been one of the basic topics in the structural analysis of the portal frame, which can provide benchmark in its structure design [1-4]. The joints in the frame were often assumed to be rigid in the theoretical analysis of the stability of the portal frame. However, a series of studies $[1,5,6]$ indicated that the joints connecting two straight members of the frame could exhibit a certain degree of flexibility. These semirigid joints may negatively affect the stability behavior of the frame. It has been pointed out that the joint flexibility should not be ignored in the design of the frame [7]. Therefore, it is necessary to adequately estimate the joint flexibility, and suitable methods considering this joint flexibility for the stability design of the frame should be established.
The flexibility of the joint can be presented by the moment-rotation relationship as shown in Figure 1. For a rigid joint, there is no relative rotation between two connected members and the rotational stiffness is infinite, while for a flexible joint, the moment $(M)$ transferred between connected members can be expressed by $M=k f\left(\theta_{r}\right)$, where $k$ is the elastic rotational spring constant, $\theta_{r}$ is angle change of the joint, and $f$ is the function of $\theta_{r}[7,8]$.

Various methods were used in the literature to study the stability of the frame with flexible joints $[2,5,10,11]$. Basaga et al. [2] combined the reliability method and finite element method to study the reliability indexes and probabilities of failure of the frame. Abu-Yasein and Frederick [10] studied the buckling capacity of a portal frame using direct stiffness method. The effects of the flexibility of the joints were considered by modifying the stiffness matrix of the frame 
elements. Wang [11] proposed a closed form solution for a symmetric trapezoidal portal frame with flexible joints and rigid members. The static balance method was used to analyze the parameters which may affect the stability of the frame. To characterize the effects of parameters on the frame stability, force and displacement curves of the frame were drawn considering these parameters. Bucking load and snap through load were defined using these load-displacement curves. Design curves were developed by drawing the buckling and snap through loads against the frame parameters. In the design curves, three status of the frame could be identified including stable, unstable, and catastrophically unstable states. Using these design curves, the shape optimization of the portal frame was made possible. This method was further extended by the author to analyze the same portal frame but with different stiffness among the joints [12]. The analysis procedure of this model was easy to follow, and the governing equation could be solved with simple solutions. However, analytical solutions by this method become impossible when asymmetric frame is considered with moving load on the frame. In order to consider more parameters and extend the application of the stability design of the portal frame, it is better to use finite element (FE) simulation. FE simulations have been used as complementary and cost-effective methods to laboratory work, as the latter could be time consuming and limited in its scope to cover extensive testing parameters [13-17].

This paper aims at the extension of the theoretical solution in $[11,12]$ by considering more parameters which may affect the stability behavior of the portal frame of a more general shape. Finite element (FE) model of an asymmetric portal frame was built with flexible joints and rigid members. This FE model was firstly validated using the closed form analytical solution in the literature. Then parameters including the position of the load, the length of the frame legs, and the span of the frame were varied and their effects on the global buckling behavior of the frame were characterized. Design curves were derived based on the FE parametric study, in which three failure modes were defined including stable, unstable, and catastrophically unstable states. Compared to the theoretical model, these FE design curves are useful for the stability analysis of the portal frame for more generalized applications. Stability optimization is made possible using the FE results so that the best shape of an asymmetric portal frame can be selected for specified applications.

\section{Finite Element Method}

2.1. Model Description. The finite element (FE) analysis software Abaqus was adopted for the simulation. Two-dimensional FE models were built for the trapezoidal frame with rigid members and flexible joints. The geometry of the portal frame is shown in Figure 2(a). The load on the frame is represented by a solid mass with a weight of $W$. The center of the mass denotes the location of the vertical load. As shown in Figure 2(a), the center of the mass is located at $\mathrm{d} L$ above the platform and $e L$ away from the center line of the platform with a length of $L$. For more general applications, the two legs of the frame are set to different lengths and slopes from the ground. The lengths and slopes of the two legs can be determined by the ratios of $h_{L}, h_{R}, c_{L}$, and $c_{R}$. The total span of the frame is equal to $\left(c_{L}+c_{R}\right) L$.

The flexible joint of the trapezoidal frame is modeled by a hinge constrained with a rotational spring. The momentrotation relationship of the joint can be represented by

$$
M=k f(\Delta \theta) \text {. }
$$

Here, $k$ is the elastic rotational spring constant. The effect of $k$ on the stability of portal frame has been studied in [12]. It concluded that, to achieve the best stability performance, it is better to have all the joints which have the same stiffness constant $k$. Therefore, $k$ was made the same for all joints in this study. $f$ means the function of the angle change $(\Delta \theta)$. For small deformation, $f(\Delta \theta)$ is a linear function, whereas the momentrotation relationship becomes nonlinear for large deformation and the joint shows a softening behavior with the increase of the rotational angle [17]. This softening behavior can be described using the following equation as proposed in [11]:

$$
f(\Delta \theta)=\frac{1}{\lambda} \tan h(\lambda \Delta \theta) .
$$

This function is bounded, monotonic, and odd in $\Delta \theta$. For small angle change, the relation between the moment and the angle change in (2) is closely linear, because the function can be expanded as follows:

$$
f(\Delta \theta) \sim \Delta \theta-\frac{\lambda^{3}}{3} \Delta \theta^{3}+O(\Delta \theta)^{5},
$$

Here, $\lambda$ reflects the nonlinearity of the spring. When $\lambda=0$, it represents linear elastic rotational spring. The true shape of the asymmetric portal frame is shown in Figure 2(a). For simplicity, the dimensions of the frame can be normalized by the length of the platform $(L)$, and the geometry of the frame in the FE simulations is shown in Figure 2(b) with the normalized load of $F=W L / k$.

When building the frame model in Abaqus, the dimension of each element was directly defined through inputting the coordinates of two ends using the GUI interface. The platform and two legs of the portal frame were meshed using discrete rigid elements (R2D2). The spring element was defined at the four joints of the frame. The sprint elements were defined through interaction function in Abaqus, in which the nonlinear relationship between rotation moment and rotation angle can be input in the interaction function. The nonlinearity as described in equation (3) was used when defining the spring element in Abaqus.

In the $F E$ analysis, the load was applied by incremental increase of the vertical displacement of the center point of the platform of the frame. This is because the displacement control would be easy to achieve convergence for buckling analysis. The vertical reaction forces of the two legs were summed up as the total load, and then the load-displacement curves were drawn based on the FE results.

2.2. Model Verification. The FE model is firstly verified using the theoretical solution reported in [11]. For the symmetric 


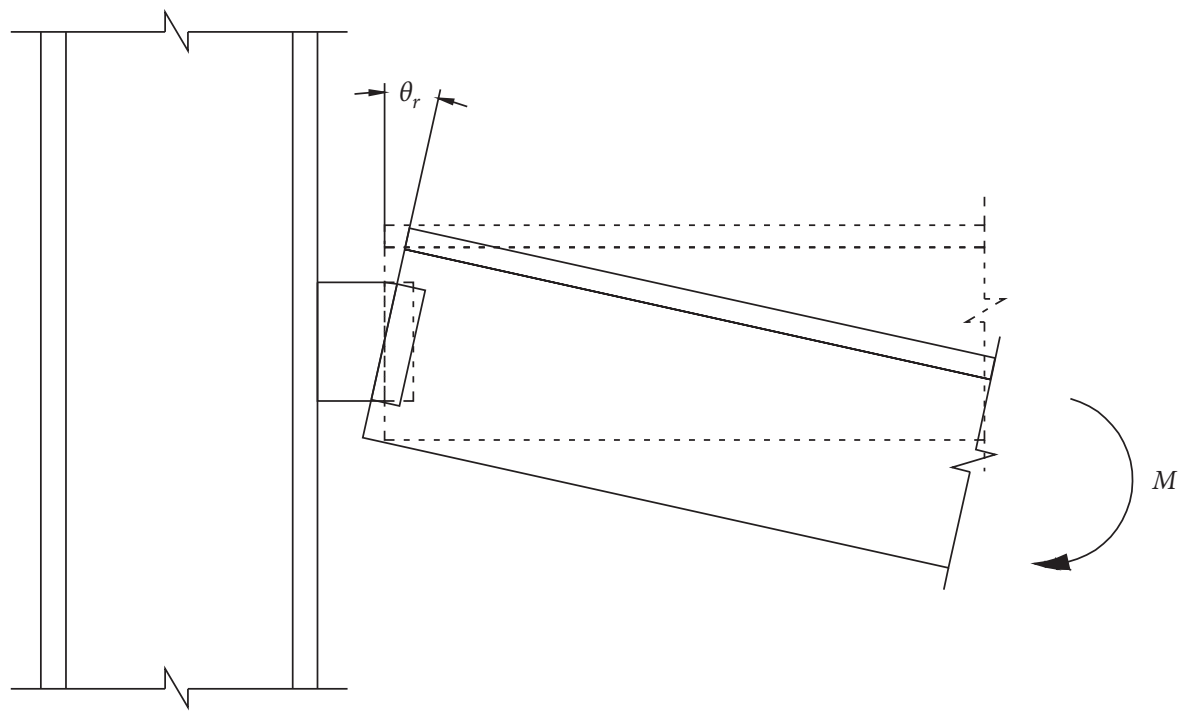

Figure 1: Rotation deformation of a connection [9].

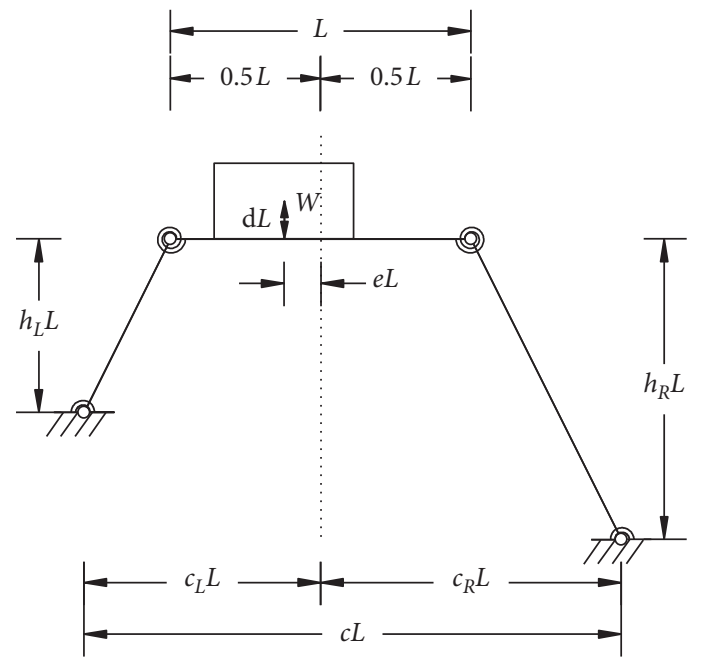

(a)
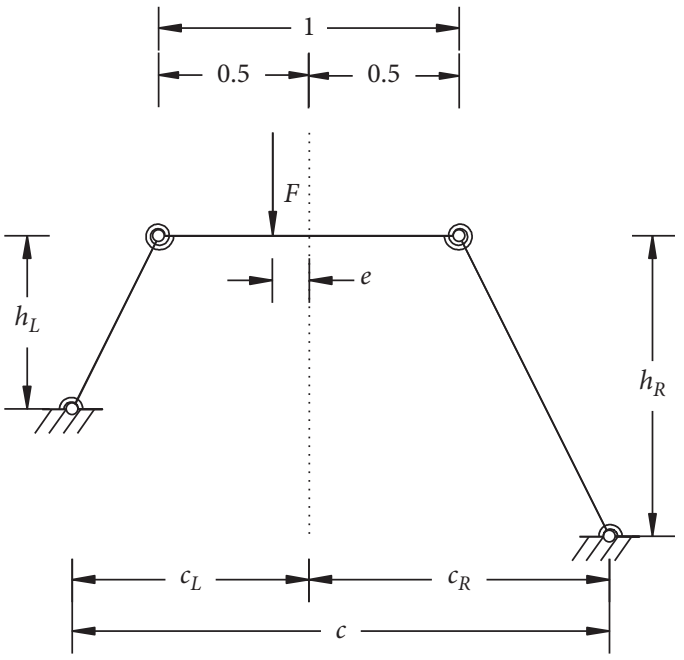

(b)

Figure 2: (a) The shape of the asymmetric trapezoidal frame with a load; (b) the geometry of the trapezoidal frame used in the FE simulations whose dimensions have been normalized.

portal frame (i.e., $h_{L}=h_{R}, c_{L}=c_{R}$, and $e=0$ in Figure 2), the load $(F)$ and displacement $(\Delta)$ relationship in the vertical direction for each selected span length $(c)$ is shown in Figure 3(a) [11]. Two loads, the buckling load $\left(F_{b}\right)$ and the snap through load $\left(F_{s}\right)$, were defined using these load-displacement curves in [11]. Taking the curve of $c=2$, for example, the load increases firstly up to condition " $A$ " before which the shape of the frame remains unchanged. Because the members of the frame are rigid, the stiffness (slope of the curve) of the loading stage " $O A$ " is infinite. If the frame is continuously loaded after point " $A$," the frame should have collapsed if the joints were pin connected. Since the flexible joints were used (see (1)), the frame can continue to carry the load by changing the shape. This process is indicated by the segment of ' $A-B-C$ ' of the curve which shows increasing load against the displacement. The load at condition " $A$ " is defined as $F_{b}$ which indicates the shape changing load. It should be noted that the frame is stable at stage " $A-B-C$," and the shape of the frame can resume when the load is removed. Point " $C$ " is the peak of the curve and the load at " $C$ " is defined as $F_{s}$. After point " $C$,' the capacity of the frame drops indicating an unstable status of the frame at the stage of " $C$ $D$-E." Therefore, $F_{s}$ is the maximum load that the frame could carry without catastrophic collapse.

As discussed above, the buckling load $\left(F_{b}\right)$ and the snap through load $\left(F_{s}\right)$ are two important characteristic loads which define the status of the frame, that is, stable and shape maintained when $F<F_{b}$, unstable with shape changing when $F_{b}<F<F_{s}$ and catastrophically unstable when $F>F_{s}$. In Wang's study [11], only symmetric portal frame was considered. Therefore, the only parameter that affects $F_{b}$ and $F_{s}$ is the span of $c$ (see Figure $3(\mathrm{a})) . F_{b}$ and $F_{s}$ were plotted against $c$ in Figure 3(b) [11]. It can be seen that the curves of $F_{b}$ and $F_{s}$ divide the graph into three regions. The region 


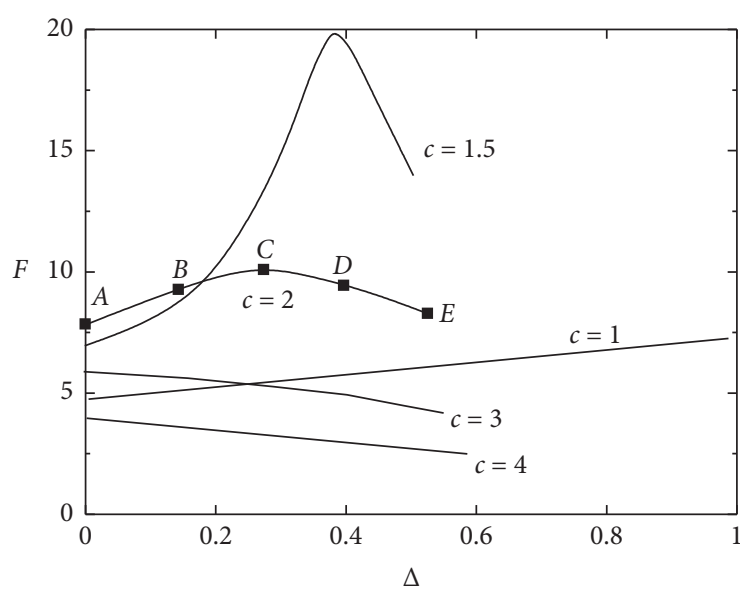

(a)

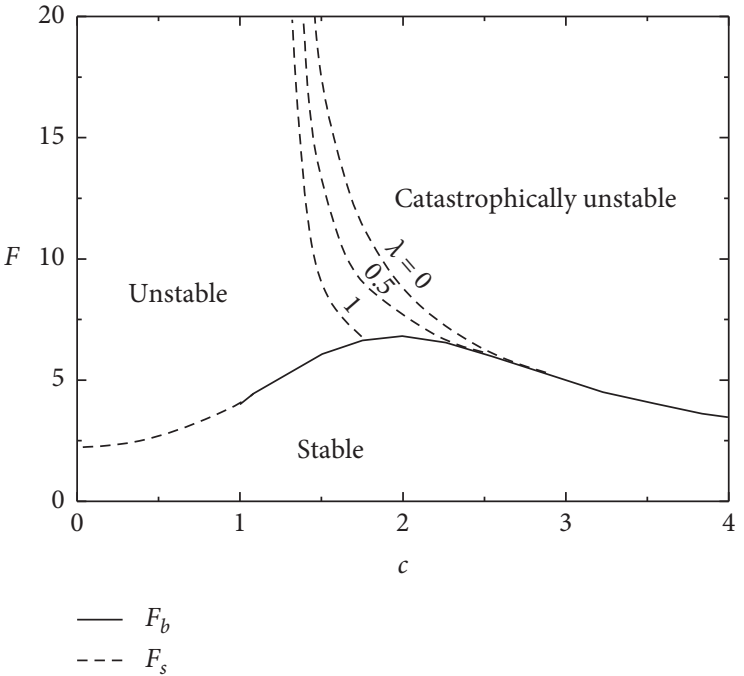

(b)

Figure 3: (a) Load-displacement curves of the symmetric portal frame with $h=1, d=0, \lambda=0$; (b) design curves of the symmetric portal frame showing the change of $F_{b}$ and $F_{s}$ against the span length of the frame $c$ [11].

under the curve of $F_{b}$ refers to the stable status of the frame, while the region above $F_{b}$ and on the left of $F_{s}$ denotes the unstable with shape changing status and the region on the right of $F_{s}$ means the frame is in the catastrophic unstable status.

The FE simulations were performed by the authors to reproduce the curves in Figure 3(b). The same symmetric portal frame as in [11] was modeled (i.e., $h_{L}=h_{R}, c_{L}=c_{R}$, and $e=0$ in Figure 2(a)) and the flexible joints were represented using equations (1)-(3). The FE curves are plotted in Figure 4. It can be seen that the FE predictions agree well with the theoretical results in Figure 3(b), indicating the validity of the FE model. Please note that the curve of $F_{b}$ is only meaningful when $c>1$, because the span of the frame $c$ is normally larger than the length of the platform in real applications.

\section{Parametric Studies and Discussion}

A more generalized asymmetric shape of the portal frame in Figure 2 was modeled in FE. A comprehensive parametric study was conducted to investigate the effects of various parameters on the buckling behavior of the frame. The considered parameters are listed in Table 1. In order to isolate and clarify the effect of each parameter on the buckling behavior of the frame, other parameters were kept constant when the specific parameter was studied.

To make the FE study more practical, several parameters were set to constant to reflect the most common applications of the portal frame. For example, the vertical distance of the load to the platform ( $d L$ in Figure 2) was set to zero, because it is usually small and negligible comparing to the other dimensions of the frame. The rotational springs at the joints of the frame were assumed to be linear with $\lambda$ in equation (3) equal to zero. As can be seen in equation (3), the nonlinearity of the springs is determined by $\lambda$. It was reported in [11] that the snap through load $\left(F_{s}\right)$ would decrease with the increase

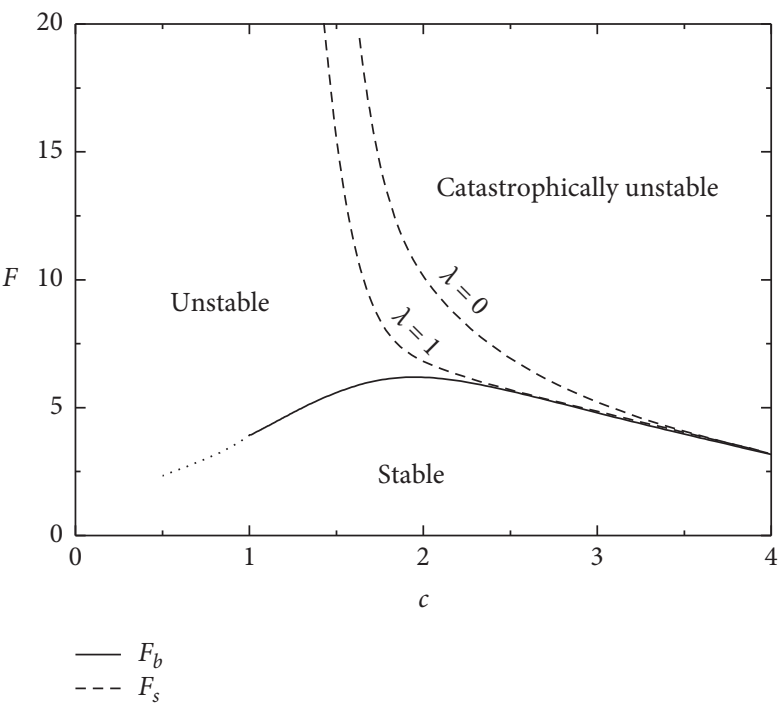

FIGURE 4: FEM result of $F_{b}$ and $F_{s}$ against the span length of the frame $c$ for a symmetric portal frame.

of $\lambda$ or nonlinearity of the springs. Therefore, it is recommended in the real applications, to design the joints with linear moment-rotation relationship $(\lambda=0)$ so that the stability of the frame can be improved. In addition, the height of the right leg was set to $h_{R}=1$ as it was used in [11].

As mentioned in the model description, the load-displacement curve of each FE model was firstly drawn, so that the buckling load $\left(F_{b}\right)$ and the snap through load $\left(F_{s}\right)$ could then be identified and extracted. Then after a serious of FE simulations with the variation of a parameter, the effects of this specific parameter on $F_{b}$ and $F_{s}$ can be achieved. $F_{b}$ and $F_{s}$ can be plotted against the geometry of the frame with the consideration of effects of various parameters, which can serve as the design curves for the stability design of an asymmetric portal frame. 
TABle 1: Variables considered in the $F E$ studies.

\begin{tabular}{lc}
\hline Parameters & Meaning \\
\hline$c_{L}$ & Normalized distance from the left leg to the center of the platform \\
$c_{R}$ & Normalized distance from the right leg to the center of the platform \\
$h_{L}$ & Normalized height of the left leg \\
$e$ & Eccentricity (distance from the load to the center of the platform) \\
\hline
\end{tabular}

\subsection{Effect of the Eccentricity of Loading (e) on the Stability of a} Symmetric Frame. The portal frame normally carries moving load on its platform. Taking the gantry crane, for example, the lifted weight is running along the platform. Therefore, it is important to understand how the eccentricity of the load $(e)$ affects the stability of the frame. In this part, a symmetric frame is considered with $c_{L}=c_{R}$ and $h_{L}=h_{R}$ in Figure 2. Three locations of the load were considered, with the values of $e$ equal to $0,0.25$, and 0.5 . $e=0$ means the load is at the center of the platform (no eccentricity). $e=0.25$ indicates the load at a quarter of the length of the platform. When the load is placed at either of the top joints in Figure 2, $e=0.5$. For the three cases, various span lengths of $c=c_{L}+c_{R}$ were simulated in the FE model and the corresponding buckling load $\left(F_{b}\right)$ and snap through load $\left(F_{s}\right)$ were obtained and plotted against $c$ in Figure 5.

Three interesting observations are found in Figure 5. When the span of the frame $c$ is fixed, with the increase of the eccentricity, the stability of the frame becomes worse with reduced $F_{b}$ and $F_{s}$. Consequently, the areas of the stable and unstable regions reduce, and the area of the catastrophic unstable region becomes larger. In other words, it is better to place the load at the center of the platform to achieve the optimum stability of the symmetric portal frame. Using the design curves in Figure 5, the corresponding $F_{b}$ and $F_{s}$ can be directly obtained for a given eccentricity of $e$.

Another interesting observation is that when $c=1$ (which means a rectangular frame with two legs perpendicular to the ground), the eccentricity seems to have little effect on the buckling load $F_{b}$. This is easy to understand because the vertical loading is completely supported by the vertical reaction forces, and no horizontal component of the reaction forces can be developed at the supports wherever the load locates given $c=1$.

It is also interesting to notice that when $c>1$, the trend of the buckling load curve without eccentricity $(e=0)$ is different from the trend of the curves with nonzero eccentricity $(e \neq 0)$. When $e=0, F_{b}$ firstly increases with the span $c$ up to a peak value, after which $F_{b}$ starts decreasing with the increase of $c$. This means there is a limit of $c$, at which the frame can reach an optimum stable state. This phenomenon was also observed by Wang in $[11,12]$. When $e \neq 0, F_{b}$ always decreases with the increase of the span $c$, indicating that the stability becomes worse with longer span. This is because the horizontal components of the reaction forces of the two legs are not equal when there is loading eccentricity. The frame will start to deform laterally as soon as the eccentric load is applied due to these unbalanced horizontal reaction forces.

3.2. Effect of the Asymmetry on the Stability of the Frame with $c_{R} \neq c_{L}$. For an asymmetric portal frame, the horizontal

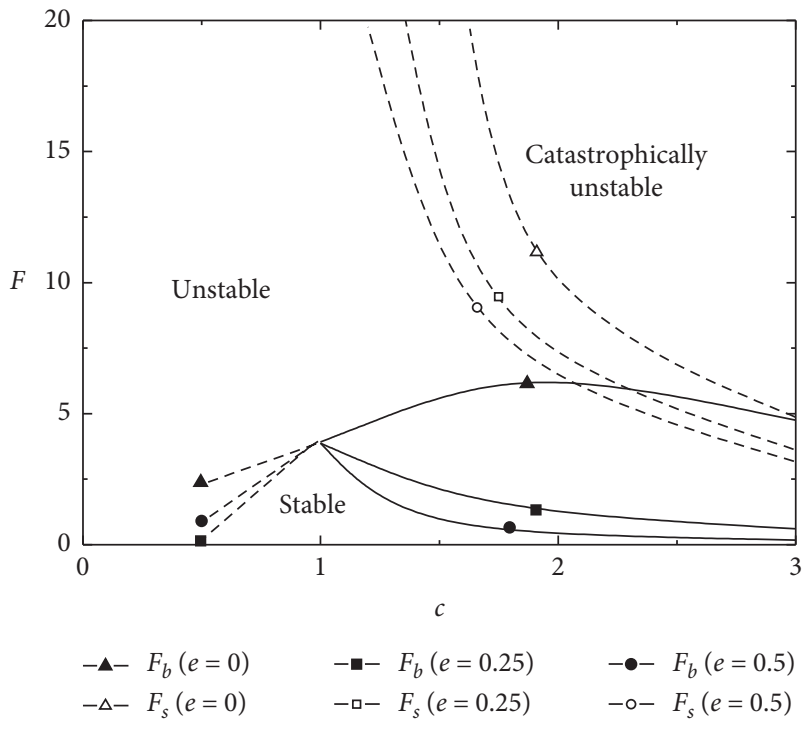

FIGURE 5: Effect of the eccentricity of loading e on the buckling load $\left(F_{b}\right)$ and snap through load $\left(F_{s}\right)$ of a symmetric portal frame.

distances from the supports of the two legs to the center of the platform $\left(c_{R}\right.$ and $\left.c_{L}\right)$ are not necessarily the same, so as to the heights of the two legs $\left(h_{R}\right.$ and $\left.h_{L}\right)$. The effect of the difference in $c_{R}$ and $c_{L}$ on the stability of the frame was firstly studied in this section considering $h_{R}=h_{L}$ and $e=0$ as shown in Figure 6.

The load-displacement curves of a portal frame with $c_{R}=h_{R}=h_{L}=1, e=0$, and various $c_{L}$ are plotted in Figure 7. It is interesting to see that when $c_{R} \neq c_{L}$, there is no buckling load $\left(F_{b}\right)$ from the load-displacement curves, while the peaks of these curves represent the corresponding snap through loads $\left(F_{s}\right)$. Only for symmetric frame with $c_{R}=c_{L}=1$, both $F_{b}$ and $F_{s}$ can be observed.

The reason for the absence of $F_{b}$ for the asymmetric frame with $c_{R} \neq c_{L}$ can be illustrated in Figure 8. Due to the asymmetry, the horizontal components of the reaction forces of the two supports are not balanced. Therefore, as soon as the load is applied, the frame will immediately deform in the horizontal direction without buckling phenomenon.

To study the effect of the asymmetry due to $c_{R} \neq c_{L}$ on the stability of the portal frame, two asymmetric conditions were selected. One condition was the left leg perpendicular to the ground with $c_{L}=0.5$, which represents the extreme condition of this asymmetry. The second condition was selected with $c_{R}>c_{L}>0.5$, showing that the left leg was in between the vertical left leg and the symmetric leg condition. In this current study, $c_{L}$ was chosen as $\left(2 c_{R}+1\right) / 4$ which satisfied the second condition. The two conditions are illustrated in 


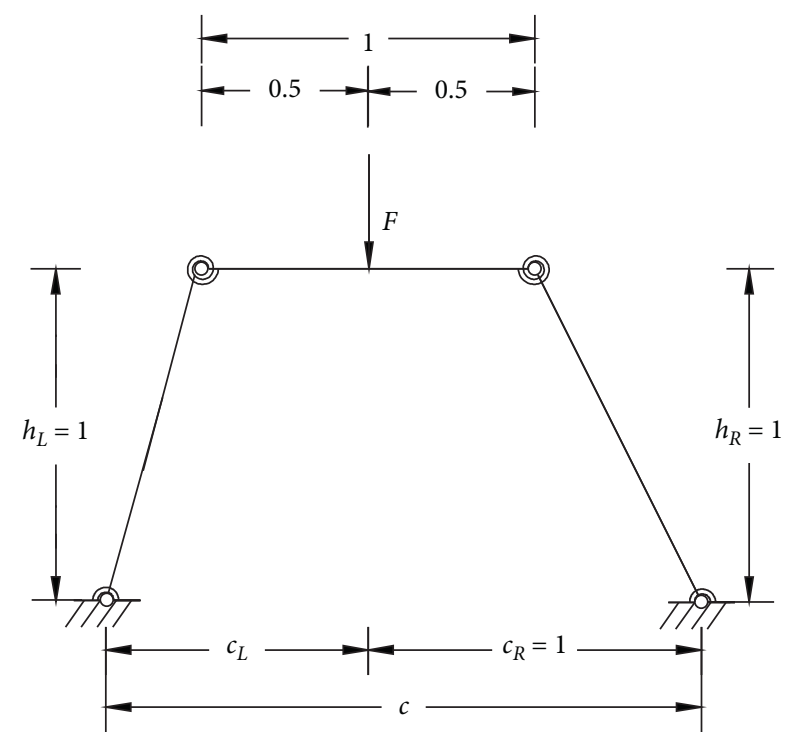

FIGURE 6: Geometry of the asymmetric portal frame adopted in the FE analysis with $c_{R} \neq c_{L}$.

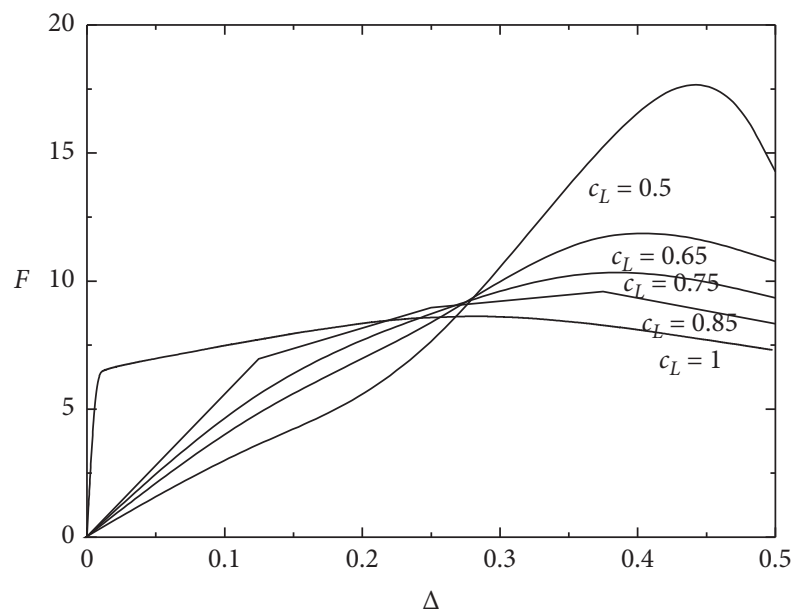

Figure 7: Load-displacement curves of a frame with $c_{R}=h_{R}=h_{L}=1, e=0$, and changing $c_{L}$.

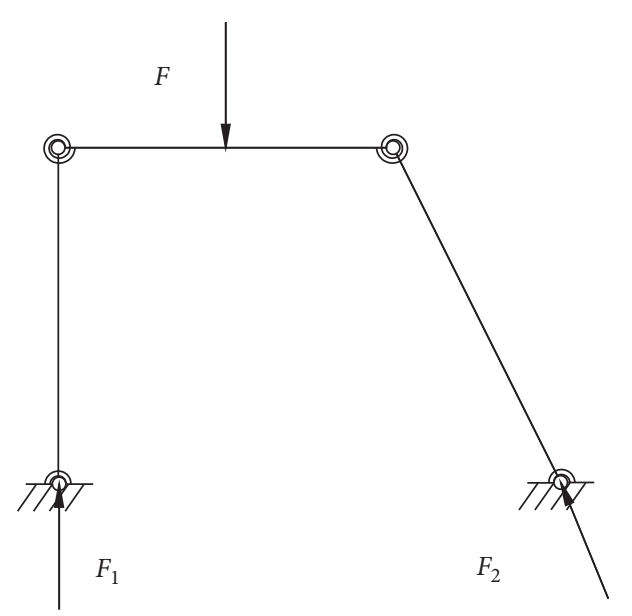

FIgURE 8: Explanation of the absence of the buckling load $F_{b}$ for a frame with $c_{R} \neq c_{L}$.
Figure 9. In the FE simulation of each condition, $c_{R}$ was changed to obtain the curves of $F_{s}$ against the span $c$ as shown in Figure 10. $F_{b}$ and $F_{s}$ curves of the symmetric condition with $c_{R}=c_{L}$ are also plotted in Figure 10 for comparison purpose.

It can be seen in Figure 10 that, with the left leg moving from the extreme asymmetric condition $\left(c_{L}=0.5\right)$ to the symmetric condition $\left(c_{L}=c_{R}\right)$, the area of the unstable with shape changing region increased with reduced catastrophic unstable region. This indicates that the larger the difference between $c_{L}$ and $c_{L R}$ is, the worse the stability of a portal frame can be. This might be why most of the portal frames are designed with a symmetric shape (i.e., $c_{L}=c_{R}$ ).

3.3. Effect of the Asymmetry on the Stability of the Frame with $h_{R} \neq h_{L}$. In the real applications of a portal frame, the heights of the left and right legs are not necessarily the same. In order to study the effect of the asymmetry of the frame due to the different heights of the legs, the height of the right leg $\left(h_{L}\right)$ was selected as a variable of the FE analysis with other parameters kept constant. For example, the distance from the right support to the center of the platform $\left(c_{R}\right)$ and the height of the right leg $\left(h_{R}\right)$ were fixed as 1 . In order to keep the same of the two angles of the left and right legs to the ground, the relationship was maintained $c_{L} / h_{L}=c_{R} / h_{R}$. The shape of the asymmetric frame with $h_{L}$ as the variable is illustrated in Figure 11(a). The eccentricity of the load is not considered $(e=0)$ and a typical deformed shape of the frame is shown in Figure 11(b).

For each specific $h_{L}$, the load-displacement curve of the frame was obtained and the corresponding buckling load $\left(F_{b}\right)$ and the snap through load $\left(F_{s}\right)$ were extracted. $F_{b}$ and $F_{s}$ are plotted against $h_{L}$ in Figure 12. It can be seen that there is a turning point for both $F_{b}$ and $F_{s}$. For example, when $h_{L}<0.3$, both $F_{b}$ and $F_{s}$ tend to be increasing very fast to infinity. On the other hand, when $h_{L}>0.3, F_{b}$ and $F_{s}$ become almost constant. This observation indicates that there exists a critical height of the left leg $\left(h_{L, c r}\right)$. It is always better to make sure that $h_{L} \leqq h_{L, c r}$ to get a more stable frame. If $h_{L}>h_{L, c r}$, the stability of the frame can hardly be affected by the change of the height of the left leg.

This critical height of the left leg $\left(h_{L, c r}\right)$ can be explained through the deformed shape of the frame. The typical deformed shape of a frame with $h_{L}<0.3$ is plotted in Figure 13(a). It can be seen that the left leg may rotate anticlockwise in this case. When the top left joint touches the ground, the deformation stops representing the final shape of the frame. This means the frame will not deform further so that the snap through load $F_{s}$ becomes infinite. One extreme condition of $h_{L}<0.3$ is $h_{L}=0$ (when there is no left leg). In this case, the shape of the frame is a triangle which has infinite $F_{b}$ and $F_{s}$. On the other hand, when $h_{L}>0.3$, the deformed shape of the frame is shown in Figure 13(b). It can be seen that, with the increase of the loading, the platform is gradually lowered and the angle of the top right joint becomes larger. When $F_{s}$ is exceeded, the angle will be larger than 180 degree indicating the inflection condition. 


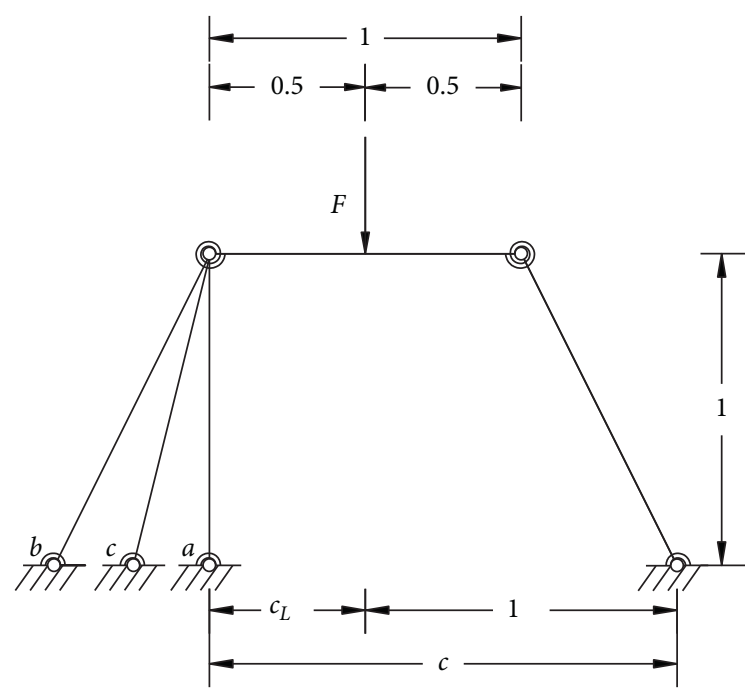

FIGURE 9: Illustration of the (a) asymmetric condition with $c_{L}=0.5$; (b) symmetric condition; and (c) asymmetric condition with $c_{L}=\left(2 c_{R}+1\right) / 4$.

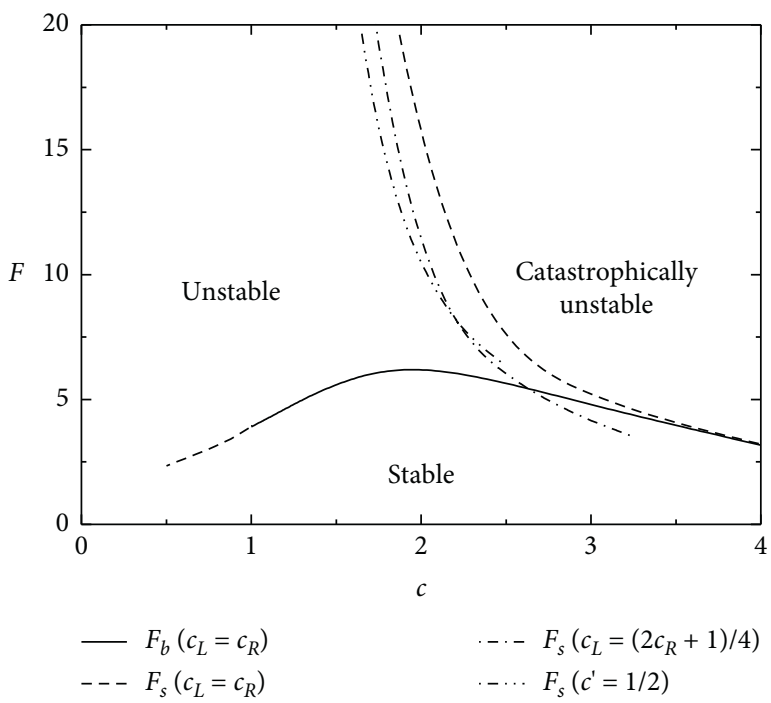

Figure 10: Effect of asymmetry $\left(c_{R} \neq c_{L}\right)$ on the snap through load $\left(F_{s}\right)$ of a portal frame with $h_{R}=h_{L}=1$ and $e=0$.

Therefore, the deformed shape of the frame is determined by the critical height of the left leg $\left(h_{L, c r}\right)$.

Actually, the above discussions on the critical height of the left leg $\left(h_{L, c r}=0.3\right)$ are based on a given shape of an asymmetric portal frame, that is, $c_{R}=h_{R}=1, e=0$, and $c_{L}$ / $h_{L}=c_{R} / h_{R}$. In fact, the value of $h_{L, c r}$ is dependent on the distance from the right support to the center of the platform $\left(c_{R}\right)$. The relationship between $h_{L, c r}$ and $c_{R}$ is obtained through a series of $F E$ analysis with changing $c_{R}$ as shown in Figure 14. It can be seen that $h_{L, c r}$ becomes smaller with the increase of $c_{R}$. This indicates that, for a given span of a frame $(c)$, it is necessary to find out the corresponding critical height of the left leg $\left(h_{L, c r}\right)$ and then make sure that the design height is smaller than $h_{L, c r}$ to achieve a better stability of the frame.

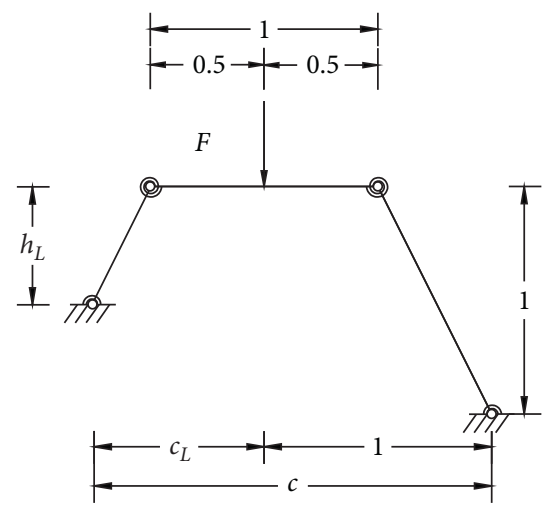

(a)

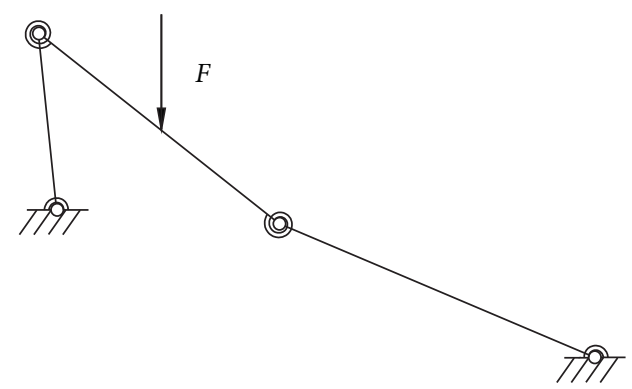

(b)

Figure 11: (a) The geometry of the asymmetric portal frame adopted in the $F E$ analysis (with $h_{R}=1, e=0$, and $c_{L} / h_{L}=c_{R} / h_{R}$ ) and (b) a typical deformed shape of the portal frame under loading.

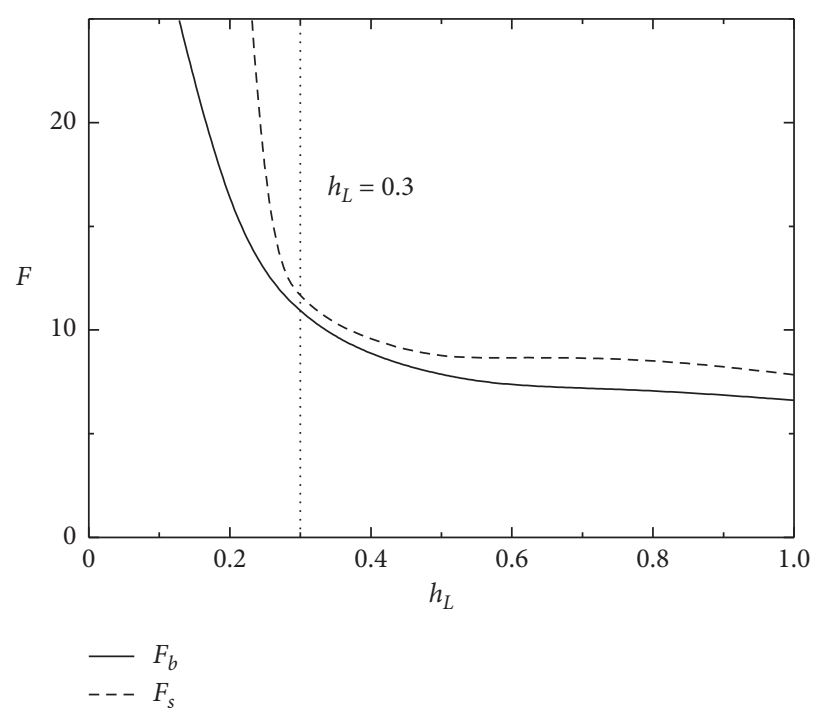

FIGURE 12: Effect of the height of the left leg $\left(h_{L}\right)$ on the buckling load $\left(F_{b}\right)$ and the snap through load $\left(F_{s}\right)$ of a portal frame with $c_{R}=h_{R}=1, e=0$, and $c_{L} / h_{L}=c_{R} / h_{R}$.

The buckling load $\left(F_{b}\right)$ and the snap through load $\left(F_{s}\right)$ of the asymmetric frame with the unequal left and right legs are plotted in Figure 15 against the span $c_{R}$. Three values of $h_{L}$ were selected including $0.2,0.6$, and 1 . It can be seen in Figure 15 that both $F_{b}$ and $F_{s}$ increase with 


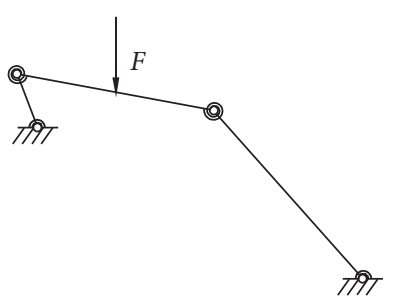

(a)

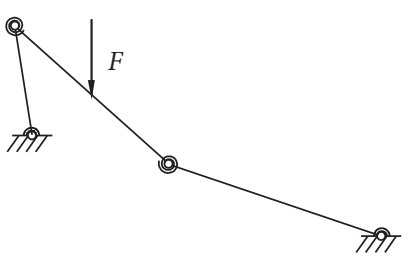

(b)

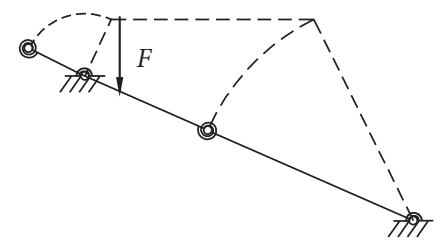

(c)

Figure 13: Typical deformed shape of an asymmetric frame when (a) $h_{L}<0.3$, (b) $h_{L}>0.3$, and (c) $h_{L}=0.3$.

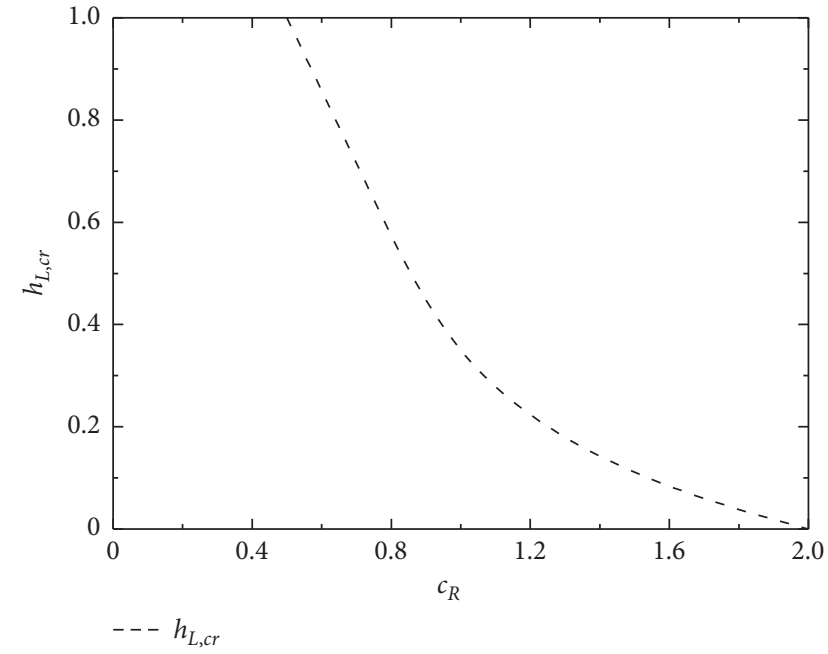

FIGURE 14: Relationship between the critical height of the left leg $\left(h_{L, c r}\right)$ and the distance from the right support to the center of the platform $c_{R}$.

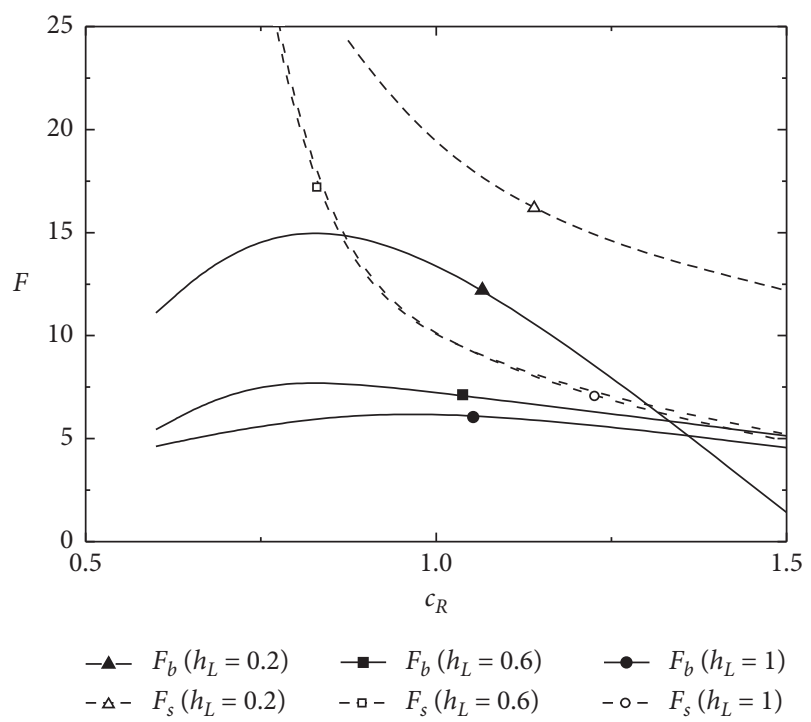

FIGURE 15: Effect of asymmetry $\left(h_{R} \neq h_{L}\right)$ on the buckling load $\left(F_{b}\right)$ and snap through load $\left(F_{s}\right)$ of a portal frame with $c_{L} / h_{L}=c_{R} / h_{R}$ and $e=0$.

smaller $h_{L}$. In addition, it is noticed that $F_{b}$ and $F_{s}$ curves of $h_{L}=0.6$ are very close to those of $h_{L}=1$. This is because when $h_{L}>h_{L, c r}, h_{L}$ has little effect on the stability of the portal frame as has already been discussed in Figure 12 .

\section{Conclusions}

This paper presents a numerical study on the stability of a trapezoidal portal frame with rigid members and flexible joints. The numerical finite element $(F E)$ model was firstly validated using the theoretical solution to the stability of a symmetric portal frame from the literature. Then the theoretical analysis in the literature was extended by considering the effects of more parameters on the stability of the portal frame of a general shape. The parameters considered include the eccentricity of the load $(e)$, the asymmetry of the frame due to the unequal distances from the left and right supports to the center of the platform $\left(c_{L} \neq c_{R}\right)$, and the asymmetry of the frame due to the unequal length of the left and right legs $\left(h_{L} \neq h_{R}\right)$. The effects of these parameters on the stability of the frame were characterized through their effects on the buckling load $\left(F_{b}\right)$ and snap through load $\left(F_{s}\right)$ of the frame. Design curves were developed which provide benchmark for the stability optimization of the portal frame of a general shape. Based on the results in the paper, the following conclusions can be made:

(1) For a symmetric portal frame, it is more stable when the load is acting at the center of the platform (with the eccentricity $e=0$ ). Its stability reduces with decreased buckling load and snap through load when the load moves away from the center of the platform (with the eccentricity $e \neq 0$ ). When there is only concentrated vertical load acting on the platform, it is suggested a symmetric portal frame with a rectangular shape be used to achieve the best stability performance (with $c=1$ ).

(2) For asymmetric portal frame with different distances from the left and right supports to the center of the platform $\left(c_{R} \neq c_{L}\right)$, the buckling load is absent from the load-displacement curves in which only the snap through load can be observed. The snap through load may decrease with the increase of the deference between the two distances of $c_{R}$ and $c_{L}$. This indicates that the frame immediately starts deforming as soon as the load is applied on the platform. Therefore, it suggests that the frame is more stable when $c_{L}=c_{R}$. If it is difficult to achieve this symmetry, it is better to ensure that the difference between $c_{R}$ and $c_{L}$ is as small as possible. 
(3) For asymmetric portal frame with different heights of the left and right legs $\left(h_{R} \neq h_{L}\right)$, there exists a critical height of the left leg $\left(h_{L, c r}\right)$. When $h_{L}<h_{L, c r}$, both $F_{b}$ and $F_{s}$ tend to increase very fast to infinity. When $h_{L}>h_{L, c r}, F_{b}$ and $F_{s}$ can hardly be affected by $h_{L}$. In addition, it was found that the values of $h_{L, c r}$ are dependent on the distance from the right support to the center of the platform $\left(c_{R}\right) . h_{L, c r}$ decreases with the increase of $c_{R}$. Therefore, it is recommended that when the span of a frame $(c)$ is predetermined, the first step of design should be the determination of $h_{L, c r}$. Then it should make sure that $h_{L}<h_{L, c r}$ to achieve a better stability of the frame. However, if it is $h_{L}>h_{L, c r}$, the design of $h_{L}$ is not important because the stability of the frame can hardly be affected by $h_{L}$.

\section{Data Availability}

The numerical data used to support the findings of this study are included within the article.

\section{Conflicts of Interest}

The authors declare that there are no conflicts of interest regarding the publication of this paper.

\section{Acknowledgments}

The first author $\mathrm{C}$. Wu acknowledges the funding by the National Natural Science Foundation of China (Grants nos. 51978025 and 51911530208). The third author L. H. Tam acknowledges the support from the National Natural Science Foundation of China (Grant no. 51808020).

\section{References}

[1] M. Kotelko, K. Kowal-Michalska, and J. Rhodes, "Stability of structures," Thin-Walled Structures, vol. 45, no. 10-11, pp. 809-960, 2007.

[2] H. B. Basaga, M. E. Kartal, and A. Bayraktar, "Reliability analysis of steel braced reinforced concrete frames with semirigid connections," International Journal of Structural Stability and Dynamics, vol. 12, no. 5, Article ID 1250037, 2012.

[3] C. S. Chang and D. H. Hodges, "Stability studies for curved beams," Journal of Mechanics of Materials and Structures, vol. 4, no. 7-8, pp. 1257-1270, 2009.

[4] C. Zhi-Hua, R. Bin, and A. Fafitis, "Axial compression stability of a crisscross section column composed of concrete-filled square steel tubes," Journal of Mechanics of Materials and Structures, vol. 4, no. 10, pp. 1787-1799, 2009.

[5] Y. Goto, S. Suzuki, and W.-F. Chen, "Stability behaviour of semi-rigid sway frames," Engineering Structures, vol. 15, no. 3, pp. 209-219, 1993.

[6] K. M. Romstad and C. V. Subramanian, "Analysis of frames with partial connection rigidity," Journal of the Structural Division, vol. 96, pp. 2283-2300, 1970.

[7] G. Morris, Stability Design of Steel Frames, CRC Press, Boca Raton, FL, USA, 1991.

[8] M. K. Elsati and R. M. Richard, "Derived moment rotation curves for partially restrained connections," Structural Engineering Review, vol. 8, no. 2-3, pp. 151-158, 1996.
[9] W. F. Chen and N. Kishi, "Semirigid steel beam-to-column connections: data base and modeling," Journal of Structural Engineering, vol. 115, no. 1, pp. 105-119, 1989.

[10] O. A. Abu-Yasein and G. R. Frederick, "Analysis of frames with semi-rigid joints," Computers \& Structures, vol. 52, no. 6, pp. 1161-1168, 1994.

[11] C. Y. Wang, "Optimum trapezoidal frame with rigid members and flexible joints," International Journal of Non-linear Mechanics, vol. 42, no. 5, pp. 760-764, 2007.

[12] C. Y. Wang, "Stability and optimization of the portal frame with rigid members and flexible joints," Journal of Engineering Mechanics, vol. 137, no. 5, pp. 366-370, 2011.

[13] F. Genna and C. Paganelli, "Force-displacement relationship in the extraction of a porcine tooth from its socket: experiments and numerical simulations," Journal of Mechanics of Materials and Structures, vol. 9, no. 5, pp. 497-514, 2014.

[14] B. L. Karihaloo and Q. Z. Xiao, "Accurate simulation of mixed-mode cohesive crack propagation in quasi-brittle structures using exact asymptotic fields in xfem: an overview," Journal of Mechanics of Materials and Structures, vol. 6, no. 1-4, pp. 267-276, 2011.

[15] L. Karlsson, A. Pahkamaa, M. Karlberg, M. Löfstrand, J. Goldak, and J. Pavasson, "Mechanics of materials and structures: a simulation-driven design approach," Journal of Mechanics of Materials and Structures, vol. 6, no. 1-4, pp. 277-301, 2011.

[16] J. A. Kolodziej, M. Mierzwiczak, and J. K. Grabski, "Computer simulation of the effective viscosity in brinkman filtration equation using the trefftz method," Journal of Mechanics of Materials and Structures, vol. 12, no. 1, pp. 93-106, 2017.

[17] M. Nowak, R. Pęcherski, M. Potoczek, and R. Śliwa, "Numerical simulations of mechanical properties of alumina foams based on computed tomography," Journal of Mechanics of Materials and Structures, vol. 12, no. 1, pp. 107-121, 2017. 